\title{
Measurement of Muon Antineutrino Oscillations with an Accelerator-Produced Off-Axis Beam
}

K. Abe, ${ }^{47}$ C. Andreopoulos, ${ }^{45,26}$ M. Antonova, ${ }^{21}$ S. Aoki,${ }^{23}$ A. Ariga, ${ }^{1}$ S. Assylbekov, ${ }^{7}$ D. Autiero, ${ }^{28}$ M. Barbi, ${ }^{39}$ G. J. Barker, ${ }^{55}$ G. Barr, ${ }^{35}$ P. Bartet-Friburg, ${ }^{36}$ M. Batkiewicz, ${ }^{12}$ F. Bay, ${ }^{10}$ V. Berardi, ${ }^{17}$ S. Berkman, ${ }^{3}$ S. Bhadra, ${ }^{59}$ A. Blondel,${ }^{11}$ S. Bolognesi, ${ }^{5}$ S. Bordoni, ${ }^{14}$ S. B. Boyd,${ }^{55}$ D. Brailsford, ${ }^{25,16}$ A. Bravar, ${ }^{11}$ C. Bronner, ${ }^{22}$ M. Buizza Avanzini ${ }^{9}$ R. G. Calland, ${ }^{22}$ S. Cao, ${ }^{24}$ J. Caravaca Rodríguez, ${ }^{14}$ S. L. Cartwright, ${ }^{43}$ R. Castillo, ${ }^{14}$ M. G. Catanesi,${ }^{17}$ A. Cervera, ${ }^{15}$ D. Cherdack, ${ }^{7}$ N. Chikuma, ${ }^{46}$ G. Christodoulou, ${ }^{26}$ A. Clifton, ${ }^{7}$ J. Coleman, ${ }^{26}$ G. Collazuol, ${ }^{19}$ L. Cremonesi ${ }^{38}$ A. Dabrowska, ${ }^{12}$ G. De Rosa, ${ }^{18}$ T. Dealtry, ${ }^{25}$ P. F. Denner, ${ }^{55}$ S. R. Dennis, ${ }^{26}$ C. Densham, ${ }^{45}$ D. Dewhurst,${ }^{35}$ F. Di Lodovico, ${ }^{38}$ S. Di Luise, ${ }^{10}$ S. Dolan, ${ }^{35}$ O. Drapier, ${ }^{9}$ K. E. Duffy, ${ }^{35}$ J. Dumarchez,${ }^{36}$ S. Dytman, ${ }^{37}$ M. Dziewiecki, ${ }^{54}$ S. Emery-Schrenk, ${ }^{5}$ A. Ereditato, ${ }^{1}$ T. Feusels, ${ }^{3}$ A. J. Finch, ${ }^{25}$ G. A. Fiorentini, ${ }^{59}$ M. Friend, ${ }^{13, \uparrow}$ Y. Fujii, ${ }^{13, \dagger}$ D. Fukuda, ${ }^{33}$ Y. Fukuda, ${ }^{30}$ A. P. Furmanski, ${ }^{55}$ V. Galymov, ${ }^{28}$ A. Garcia,${ }^{14}$ S. G. Giffin, ${ }^{39}$ C. Giganti, ${ }^{36}$ F. Gizzarelli, ${ }^{5}$ M. Gonin, ${ }^{9}$

N. Grant, ${ }^{25}$ D. R. Hadley, ${ }^{55}$ L. Haegel, ${ }^{11}$ M. D. Haigh, ${ }^{55}$ P. Hamilton, ${ }^{16}$ D. Hansen, ${ }^{37}$ T. Hara, ${ }^{23}$ M. Hartz,${ }^{22,51}$

T. Hasegawa, ${ }^{13, \dagger}$ N. C. Hastings, ${ }^{39}$ T. Hayashino, ${ }^{24}$ Y. Hayato, ${ }^{47,22}$ R. L. Helmer, ${ }^{51}$ M. Hierholzer, ${ }^{1}$ A. Hillairet, ${ }^{52}$ A. Himmel, ${ }^{8}$ T. Hiraki, ${ }^{24}$ S. Hirota, ${ }^{24}$ M. Hogan, ${ }^{7}$ J. Holeczek, ${ }^{44}$ S. Horikawa, ${ }^{10}$ F. Hosomi,${ }^{46}$ K. Huang,${ }^{24}$ A. K. Ichikawa, ${ }^{24}$ K. Ieki, ${ }^{24}$ M. Ikeda ${ }^{47}$ J. Imber, ${ }^{9}$ J. Insler, ${ }^{27}$ R. A. Intonti, ${ }^{17}$ T. J. Irvine, ${ }^{48}$ T. Ishida,${ }^{13, \dagger}$ T. Ishii, ${ }^{13,}$ E. Iwai, ${ }^{13}$ K. Iwamoto, ${ }^{40}$ A. Izmaylov, ${ }^{15,21}$ A. Jacob, ${ }^{35}$ B. Jamieson,${ }^{57}$ M. Jiang, ${ }^{24}$ S. Johnson, ${ }^{6}$ J. H. Jo ${ }^{32}$ P. Jonsson, ${ }^{16}$ C. K. Jung, ${ }^{32, \$}$ M. Kabirnezhad, ${ }^{31}$ A. C. Kaboth, ${ }^{41,45}$ T. Kajita, ${ }^{48,}$ H. Kakuno, ${ }^{49}$ J. Kameda, ${ }^{47}$ D. Karlen, ${ }^{52,51}$ I. Karpikov, ${ }^{21}$ T. Katori, ${ }^{38}$ E. Kearns, ${ }^{2,22, \$}$ M. Khabibullin, ${ }^{21}$ A. Khotjantsev, ${ }^{21}$ D. Kielczewska, ${ }^{53,{ }^{*}}$ T. Kikawa, ${ }^{24}$ H. Kim, ${ }^{34}$ J. Kim, ${ }^{3}$ S. King, ${ }^{38}$ J. Kisiel, ${ }^{44}$ A. Knight, ${ }^{55}$ A. Knox ${ }^{25}$ T. Kobayashi, ${ }^{13, \dagger}$ L. Koch,${ }^{42}$ T. Koga ${ }^{46}$ A. Konaka, ${ }^{51}$ K. Kondo ${ }^{24}$ A. Kopylov, ${ }^{21}$ L. L. Kormos, ${ }^{25}$ A. Korzenev, ${ }^{11}$ Y. Koshio, ${ }^{33,5}$ W. Kropp, ${ }^{4}$ Y. Kudenko, ${ }^{21,8}$ R. Kurjata, ${ }^{54}$ T. Kutter, ${ }^{27}$ J. Lagoda, ${ }^{31}$ I. Lamont, ${ }^{25}$ E. Larkin, ${ }^{55}$ M. Laveder,${ }^{19}$ M. Lawe, ${ }^{25}$ M. Lazos, ${ }^{26}$ T. Lindner,${ }^{51}$ Z. J. Liptak, ${ }^{6}$ R. P. Litchfield, ${ }^{55}$ X. Li, ${ }^{32}$ A. Longhin ${ }^{19}$ J. P. Lopez, ${ }^{6}$ L. Ludovici, ${ }^{20}$ X. Lu, ${ }^{35}$ L. Magaletti, ${ }^{17}$ K. Mahn,${ }^{29}$ M. Malek ${ }^{43}$ S. Manly, ${ }^{40}$ A. D. Marino, ${ }^{6}$ J. Marteau, ${ }^{28}$ J. F. Martin, ${ }^{50}$ P. Martins,${ }^{38}$ S. Martynenko, ${ }^{32}$ T. Maruyama,,${ }^{13 \dagger}$ V. Matveev,${ }^{21}$ K. Mavrokoridis, ${ }^{26}$ W. Y. Ma, ${ }^{16}$

E. Mazzucato ${ }^{5}$ M. McCarthy, ${ }^{59}$ N. McCauley, ${ }^{26}$ K. S. McFarland,${ }^{40}$ C. McGrew,${ }^{32}$ A. Mefodiev,${ }^{21}$ M. Mezzetto, ${ }^{19}$ P. Mijakowski, ${ }^{31}$ A. Minamino, ${ }^{24}$ O. Mineev ${ }^{21}$ S. Mine, ${ }^{4}$ A. Missert, ${ }^{6}$ M. Miura, ${ }^{47}$ S. Moriyama, ${ }^{47}$, Th. A. Mueller, ${ }^{9}$ S. Murphy, ${ }^{10}$ J. Myslik, ${ }^{52}$ T. Nakadaira, ${ }^{13, \dagger}$ M. Nakahata, ${ }^{47,22}$ K. G. Nakamura, ${ }^{24}$ K. Nakamura, ${ }^{22,13, \dagger}$ K. D. Nakamura, ${ }^{24}$ S. Nakayama, ${ }^{47, \$}$ T. Nakaya, ${ }^{24,22}$ K. Nakayoshi, ${ }^{13, \dagger}$ C. Nantais, ${ }^{3}$ C. Nielsen, ${ }^{3}$ M. Nirkko, ${ }^{1}$ K. Nishikawa, ${ }^{13, \dagger}$ Y. Nishimura, ${ }^{48}$ J. Nowak, ${ }^{25}$ H. M. O'Keeffe, ${ }^{25}$ R. Ohta, ${ }^{13, \dagger}$ K. Okumura, ${ }^{48,22}$ T. Okusawa, ${ }^{34}$ W. Oryszczak, ${ }^{53}$ S. M. Oser, ${ }^{3}$

T. Ovsyannikova, ${ }^{21}$ R. A. Owen, ${ }^{38}$ Y. Oyama, ${ }^{13, \dagger}$ V. Palladino, ${ }^{18}$ J. L. Palomino,${ }^{32}$ V. Paolone, ${ }^{37}$ N. D. Patel, ${ }^{24}$ M. Pavin,${ }^{36}$ D. Payne, ${ }^{26}$ J. D. Perkin, ${ }^{43}$ Y. Petrov, ${ }^{3}$ L. Pickard,${ }^{43}$ L. Pickering, ${ }^{16}$ E. S. Pinzon Guerra, ${ }^{59}$ C. Pistillo, ${ }^{1}$ B. Popov, ${ }^{36, \|}$ M. Posiadala-Zezula, ${ }^{53}$ J.-M. Poutissou, ${ }^{51}$ R. Poutissou, ${ }^{51}$ P. Przewlocki, ${ }^{31}$ B. Quilain, ${ }^{24}$ E. Radicioni, ${ }^{17}$ P. N. Ratoff, ${ }^{25}$ M. Ravonel, ${ }^{11}$ M. A. M. Rayner, ${ }^{11}$ A. Redij, ${ }^{1}$ E. Reinherz-Aronis, ${ }^{7}$ C. Riccio, ${ }^{18}$ P. Rojas, ${ }^{7}$ E. Rondio, ${ }^{31}$ S. Roth, ${ }^{42}$ A. Rubbia ${ }^{10}$ A. Rychter, ${ }^{54}$ R. Sacco, ${ }^{38}$ K. Sakashita,,${ }^{13 \dagger}$ F. Sánchez,${ }^{14}$ F. Sato, ${ }^{13}$ E. Scantamburlo, ${ }^{11}$ K. Scholberg, ${ }^{8, \$}$ S. Schoppmann, ${ }^{42}$ J. Schwehr, ${ }^{7}$ M. Scott, ${ }^{51}$ Y. Seiya, ${ }^{34}$ T. Sekiguchi, ${ }^{13, \dagger}$ H. Sekiya, ${ }^{47,22, \sharp}$ D. Sgalaberna, ${ }^{10}$ R. Shah,,${ }^{45,35}$ A. Shaikhiev, ${ }^{21}$ F. Shaker,${ }^{57}$ D. Shaw, ${ }^{25}$ M. Shiozawa, ${ }^{47,22}$ T. Shirahige, ${ }^{33}$ S. Short, ${ }^{38}$ M. Smy, ${ }^{4}$ J. T. Sobczyk, ${ }^{58}$ M. Sorel, ${ }^{15}$

L. Southwell, ${ }^{25}$ P. Stamoulis, ${ }^{15}$ J. Steinmann,${ }^{42}$ T. Stewart, ${ }^{45}$ Y. Suda,${ }^{46}$ S. Suvorov,${ }^{21}$ A. Suzuki, ${ }^{23}$ K. Suzuki, ${ }^{24}$ S. Y. Suzuki, ${ }^{13, \dagger}$ Y. Suzuki, ${ }^{22}$ R. Tacik,${ }^{39,51}$ M. Tada ${ }^{13, \dagger}$ S. Takahashi, ${ }^{24}$ A. Takeda,${ }^{47}$ Y. Takeuchi, ${ }^{23,22}$ H. K. Tanaka, ${ }^{47, *}$ H. A. Tanaka, ${ }^{50,51,{ }^{ף}}$ D. Terhorst, ${ }^{42}$ R. Terri, ${ }^{38}$ T. Thakore, ${ }^{27}$ L. F. Thompson ${ }^{43}$ S. Tobayama, ${ }^{3}$ W. Toki, ${ }^{7}$ T. Tomura, ${ }^{47}$ C. Touramanis, ${ }^{26}$ T. Tsukamoto, ${ }^{13, \dagger}$ M. Tzanov, ${ }^{27}$ Y. Uchida, ${ }^{16}$ A. Vacheret,${ }^{35}$ M. Vagins,${ }^{22,4}$ Z. Vallari, ${ }^{32}$ G. Vasseur, ${ }^{5}$

T. Wachala, ${ }^{12}$ K. Wakamatsu, ${ }^{34}$ C. W. Walter, ${ }^{8, *}$ D. Wark, ${ }^{45,35}$ W. Warzycha, ${ }^{53}$ M. O. Wascko, ${ }^{16,13}$ A. Weber, ${ }^{45,35}$ R. Wendell, ${ }^{24, \$}$ R. J. Wilkes, ${ }^{56}$ M. J. Wilking, ${ }^{32}$ C. Wilkinson, ${ }^{1}$ J. R. Wilson, ${ }^{38}$ R. J. Wilson, ${ }^{7}$ Y. Yamada, ${ }^{13, \dagger}$ K. Yamamoto, ${ }^{34}$ M. Yamamoto, ${ }^{24} \mathrm{C}$. Yanagisawa, ${ }^{32, * *}$ T. Yano, ${ }^{23} \mathrm{~S}$. Yen, ${ }^{51} \mathrm{~N}$. Yershov, ${ }^{21} \mathrm{M}$. Yokoyama, ${ }^{46, \sharp} \mathrm{J}$. Yoo, ${ }^{27} \mathrm{~K}$. Yoshida, ${ }^{24} \mathrm{~T}$. Yuan, ${ }^{6}$ M. Yu, ${ }^{59}$ A. Zalewska, ${ }^{12}$ J. Zalipska, ${ }^{31}$ L. Zambelli, ${ }^{13, \dagger}$ K. Zaremba, ${ }^{54}$ M. Ziembicki, ${ }^{54}$ E. D. Zimmerman, ${ }^{6}$ M. Zito, ${ }^{5}$ and J. Żmuda ${ }^{58}$

(T2K Collaboration)

${ }^{1}$ University of Bern, Albert Einstein Center for Fundamental Physics, Laboratory for High Energy Physics (LHEP), Bern, Switzerland ${ }^{2}$ Boston University, Department of Physics, Boston, Massachusetts, USA 
${ }^{3}$ University of British Columbia, Department of Physics and Astronomy, Vancouver, British Columbia, Canada

${ }^{4}$ University of California, Irvine, Department of Physics and Astronomy, Irvine, California, USA

${ }^{5}$ IRFU, CEA Saclay, Gif-sur-Yvette, France

${ }^{6}$ University of Colorado at Boulder, Department of Physics, Boulder, Colorado, USA

${ }^{7}$ Colorado State University, Department of Physics, Fort Collins, Colorado, USA

${ }^{8}$ Duke University, Department of Physics, Durham, North Carolina, USA

${ }^{9}$ Ecole Polytechnique, IN2P3-CNRS, Laboratoire Leprince-Ringuet, Palaiseau, France

${ }^{10}$ ETH Zurich, Institute for Particle Physics, Zurich, Switzerland

${ }^{11}$ University of Geneva, Section de Physique, DPNC, Geneva, Switzerland

${ }^{12} \mathrm{H}$. Niewodniczanski Institute of Nuclear Physics PAN, Cracow, Poland

${ }^{13}$ High Energy Accelerator Research Organization (KEK), Tsukuba, Ibaraki, Japan

${ }^{14}$ Institut de Fisica d'Altes Energies (IFAE), Barcelona Institute of Science and Technology, Campus UAB, Bellaterra (Barcelona), Spain

${ }^{15}$ IFIC (CSIC and University of Valencia), Valencia, Spain

${ }^{16}$ Imperial College London, Department of Physics, London, United Kingdom

${ }^{17}$ INFN Sezione di Bari and Università e Politecnico di Bari, Dipartimento Interuniversitario di Fisica, Bari, Italy

${ }^{18}$ INFN Sezione di Napoli and Università di Napoli, Dipartimento di Fisica, Napoli, Italy

${ }^{19}$ INFN Sezione di Padova and Università di Padova, Dipartimento di Fisica, Padova, Italy

${ }^{20}$ INFN Sezione di Roma and Università di Roma "La Sapienza," Roma, Italy

${ }^{21}$ Institute for Nuclear Research of the Russian Academy of Sciences, Moscow, Russia

${ }^{22}$ Kavli Institute for the Physics and Mathematics of the Universe (WPI), University of Tokyo Institutes for Advanced Study, University of Tokyo, Kashiwa, Chiba, Japan

${ }^{23}$ Kobe University, Kobe, Japan

${ }^{24}$ Kyoto University, Department of Physics, Kyoto, Japan

${ }^{25}$ Lancaster University, Physics Department, Lancaster, United Kingdom

${ }^{26}$ University of Liverpool, Department of Physics, Liverpool, United Kingdom

${ }^{27}$ Louisiana State University, Department of Physics and Astronomy, Baton Rouge, Louisiana, USA

${ }^{28}$ Université de Lyon, Université Claude Bernard Lyon 1, IPN Lyon (IN2P3), Villeurbanne, France

${ }^{29}$ Michigan State University, Department of Physics and Astronomy, East Lansing, Michigan, USA

${ }^{30}$ Miyagi University of Education, Department of Physics, Sendai, Japan

${ }^{31}$ National Centre for Nuclear Research, Warsaw, Poland

${ }^{32}$ State University of New York at Stony Brook, Department of Physics and Astronomy, Stony Brook, New York, USA

${ }^{33}$ Okayama University, Department of Physics, Okayama, Japan

${ }^{34}$ Osaka City University, Department of Physics, Osaka, Japan

${ }^{35}$ Oxford University, Department of Physics, Oxford, United Kingdom

${ }^{36}$ UPMC, Université Paris Diderot, CNRS/IN2P3, Laboratoire de Physique Nucléaire et de Hautes Energies (LPNHE), Paris, France

${ }^{37}$ University of Pittsburgh, Department of Physics and Astronomy, Pittsburgh, Pennsylvania, USA

${ }^{38}$ Queen Mary University of London, School of Physics and Astronomy, London, United Kingdom

${ }^{39}$ University of Regina, Department of Physics, Regina, Saskatchewan, Canada

${ }^{40}$ University of Rochester, Department of Physics and Astronomy, Rochester, New York, USA

${ }^{41}$ Royal Holloway University of London, Department of Physics, Egham, Surrey, United Kingdom

${ }^{42}$ RWTH Aachen University, III. Physikalisches Institut, Aachen, Germany

${ }^{43}$ University of Sheffield, Department of Physics and Astronomy, Sheffield, United Kingdom

${ }^{44}$ University of Silesia, Institute of Physics, Katowice, Poland

${ }^{45}$ STFC, Rutherford Appleton Laboratory, Harwell Oxford, and Daresbury Laboratory,

Warrington, United Kingdom

${ }^{46}$ University of Tokyo, Department of Physics, Tokyo, Japan

${ }^{47}$ University of Tokyo, Institute for Cosmic Ray Research, Kamioka Observatory, Kamioka, Japan

${ }^{48}$ University of Tokyo, Institute for Cosmic Ray Research, Research Center for Cosmic Neutrinos, Kashiwa, Japan

${ }^{49}$ Tokyo Metropolitan University, Department of Physics, Tokyo, Japan

${ }^{50}$ University of Toronto, Department of Physics, Toronto, Ontario, Canada

${ }^{51}$ TRIUMF, Vancouver, British Columbia, Canada

${ }^{52}$ University of Victoria, Department of Physics and Astronomy, Victoria, British Columbia, Canada

${ }^{53}$ University of Warsaw, Faculty of Physics, Warsaw, Poland

${ }^{54}$ Warsaw University of Technology, Institute of Radioelectronics, Warsaw, Poland

${ }^{55}$ University of Warwick, Department of Physics, Coventry, United Kingdom 


\footnotetext{
${ }^{56}$ University of Washington, Department of Physics, Seattle, Washington, USA

${ }^{57}$ University of Winnipeg, Department of Physics, Winnipeg, Manitoba, Canada

${ }^{58}$ Wroclaw University, Faculty of Physics and Astronomy, Wroclaw, Poland

${ }^{59}$ York University, Department of Physics and Astronomy, Toronto, Ontario, Canada
}

(Received 8 December 2015; published 5 May 2016)

\begin{abstract}
T2K reports its first measurements of the parameters governing the disappearance of $\bar{\nu}_{\mu}$ in an off-axis beam due to flavor change induced by neutrino oscillations. The quasimonochromatic $\bar{\nu}_{\mu}$ beam, produced with a peak energy of $0.6 \mathrm{GeV}$ at J-PARC, is observed at the far detector Super-Kamiokande, $295 \mathrm{~km}$ away, where the $\bar{\nu}_{\mu}$ survival probability is expected to be minimal. Using a data set corresponding to $4.01 \times 10^{20}$ protons on target, 34 fully contained $\mu$-like events were observed. The best-fit oscillation parameters are $\sin ^{2}\left(\bar{\theta}_{23}\right)=0.45$ and $\left|\Delta \bar{m}_{32}^{2}\right|=2.51 \times 10^{-3} \mathrm{eV}^{2}$ with $68 \%$ confidence intervals of $0.38-0.64$ and $2.26-2.80 \times 10^{-3} \mathrm{eV}^{2}$, respectively. These results are in agreement with existing antineutrino parameter measurements and also with the $\nu_{\mu}$ disappearance parameters measured by $\mathrm{T} 2 \mathrm{~K}$.
\end{abstract}

DOI: 10.1103/PhysRevLett.116.181801

Introduction.-In the three-flavor framework, neutrino oscillation can be described by the unitary PontecorvoMaki-Nakagawa-Sakata matrix, which is parameterized by three angles $\theta_{12}, \theta_{23}, \theta_{13}$ and a $C P$-violating phase $\delta_{C P}$ [1-3]. Given a neutrino propagation distance, $L(\mathrm{~km})$, and energy, $E_{\nu}(\mathrm{GeV})$, such that $L / E_{\nu} \sim O(1000)$, the survival probability for a muon neutrino propagating through vacuum can be approximated by

$$
\begin{aligned}
P\left(\nu_{\mu} \rightarrow \nu_{\mu}\right) \simeq & 1-4 \cos ^{2}\left(\theta_{13}\right) \sin ^{2}\left(\theta_{23}\right) \\
& \times\left[1-\cos ^{2}\left(\theta_{13}\right) \sin ^{2}\left(\theta_{23}\right)\right] \sin ^{2}\left(\frac{1.267 \Delta m_{32}^{2} L}{E_{\nu}}\right),
\end{aligned}
$$

where $\Delta m_{32}^{2}\left(\mathrm{eV}^{2}\right)$ is the neutrino mass squared splitting, defined as $m_{3}^{2}-m_{2}^{2}$. Equation (1) shows that measuring the disappearance probability as a function of $L / E_{\nu}$ leads to a measurement of the oscillation parameters. In this model of neutrino oscillation, the disappearance probability in vacuum is identical for neutrinos and antineutrinos. The disappearance probabilities in matter can differ by as much as $0.1 \%$ for the $\mathrm{T} 2 \mathrm{~K}$ baseline and neutrino flux, but our data set is not sensitive to this small effect. Observing a significant difference between the disappearance probabilities of neutrinos and antineutrinos would, therefore, be evidence for new physics [3]. Results from the MINOS [4] and Super-Kamiokande (SK) Collaborations [5] indicate no significant difference between muon antineutrino oscillations and muon neutrino oscillations.

In this Letter, we present the first measurement of $P\left(\bar{\nu}_{\mu} \rightarrow \bar{\nu}_{\mu}\right)$ by the T2K Collaboration. This analysis allows

Published by the American Physical Society under the terms of the Creative Commons Attribution 3.0 License. Further distribution of this work must maintain attribution to the author(s) and the published article's title, journal citation, and DOI. the dominant antineutrino oscillation parameters for $\bar{\nu}_{\mu}$ disappearance to vary independently from those describing neutrino oscillations, i.e., $\theta_{23} \neq \bar{\theta}_{23}$ and $\Delta m_{32}^{2} \neq \Delta \bar{m}_{32}^{2}$, where the barred parameters refer to antineutrino oscillations. $\bar{\theta}_{13}, \bar{\theta}_{12}$, and $\Delta \bar{m}_{21}^{2}$ are assumed to be identical to their matter counterparts since our data set cannot constrain them. This ensures that the expected background at the far detector is consistent with the current knowledge of neutrino oscillations, while allowing us to use the T2K antineutrino-mode data to measure $\bar{\theta}_{23}$ and $\Delta \bar{m}_{32}^{2}$.

$T 2 \mathrm{~K}$ experiment.-The $\mathrm{T} 2 \mathrm{~K}$ experiment $[6]$ is composed of a neutrino beam line, a suite of near detectors, and the far detector, Super-Kamiokande. Both the far detector and one of the near detectors are placed $2.5^{\circ}$ off the neutrino beam axis and so observe a narrow-band beam [7]. This "off-axis" method reduces backgrounds from higher-energy neutrinos, producing a neutrino flux that peaks around $0.6 \mathrm{GeV}$, the energy at which the first minimum in the $\bar{\nu}_{\mu}$ survival probability is expected to occur at the $\mathrm{T} 2 \mathrm{~K}$ baseline.

The J-PARC main ring provides a $30-\mathrm{GeV}$ proton beam which impinges upon a graphite target, producing pions and kaons. The target is held inside the first of three magnetic horns which focus charged particles into a 96-m-long decay volume, where they decay and produce neutrinos. The polarity of the horn current determines whether positive or negative mesons are focused, which in turn determines whether the neutrino beam is largely composed of muon neutrinos or muon antineutrinos. The decay volume ends in a beam dump followed by the muon monitor, which measures the neutrino beam direction on a bunch-by-bunch basis using muons from the meson decays.

The near-detector complex [6] consists of the on-axis Interactive Neutrino GRID detector (INGRID) [8] and the off-axis detector (ND280), both $280 \mathrm{~m}$ downstream of the proton-beam target. INGRID is a $7+7$ array of ironscintillator detectors, arranged in a "cross" configuration at the beam center. INGRID provides high-statistics monitoring of the neutrino beam intensity, direction, profile, and 
stability and has shown that the neutrino beam direction is controlled to $0.4 \mathrm{mrad}$. ND280 consists of a number of subdetectors installed inside the refurbished UA1/NOMAD magnet, which provides a $0.2 \mathrm{~T}$ field. The near-detector analysis described here uses the tracker region of ND280, which consists of three time projection chambers (TPC1, 2, 3) [9] interleaved with two fine-grained detectors (FGD1, 2) [10]. The FGDs are the neutrino target and track charged particles coming from the interaction vertex, while the TPCs perform 3D tracking and determine the charge, momentum, and energy loss of each charged particle traversing them. The observed energy loss is used for particle identification which, when combined with particle charge information, allows a precise separation and measurement of the $\bar{\nu}_{\mu}$ (right-sign) and $\nu_{\mu}$ (wrong-sign) interactions in the antineutrino-mode beam.

The far detector is a 50-kt (22.5-kt fiducial mass) water Cherenkov detector $[11,12]$, where the volume is divided into an outer detector (OD) with 1885 outward-facing 20-cm-diameter photomultiplier tubes and an inner detector (ID) with 11129 inward-facing 50-cm-diameter photomultiplier tubes. The events arriving at SK from the J-PARC beam spill are synchronized with a global positioning system with $<150 \mathrm{~ns}$ precision.

The results presented here are based on data taken in three periods: two where the beam operated in antineutrino mode, (1) June 2014 and (2) November 2014-June 2015, and one in neutrino mode, (3) November 2010-May 2013. The oscillation analysis uses periods (1) and (2), while the near-detector analysis uses data from periods (1) and (3). This corresponds to an exposure of $4.01 \times 10^{20}$ protons on target (POT) in antineutrino mode for the oscillation analysis, and an exposure of $0.43 \times 10^{20}$ POT in antineutrino mode plus $5.82 \times 10^{20} \mathrm{POT}$ in neutrino mode for the near-detector analysis.

Analysis strategy.-This analysis resembles that of Ref. [13], fitting samples of charged-current (CC) interactions at ND280 to produce a tuned prediction of the unoscillated antineutrino spectrum at the far detector, including its associated uncertainty. This analysis differs from Ref. [13] in that both $\nu_{\mu}$ and $\bar{\nu}_{\mu}$ samples at ND280 are fitted. This ensures that the neutrino interaction model is consistent between both neutrino- and antineutrino-beammode data sets and provides a constraint on both the right-sign signal and the wrong-sign background in the antineutrino-mode beam.

Flux simulation.-The nominal neutrino flux at ND280 and SK (without oscillation) is predicted by simulating the secondary beam line [14] using FLUKA2011 $[15,16]$ and GEANT3 with GCALOR $[17,18]$. The simulated hadronic interactions are tuned to external hadron-production data. The unoscillated neutrino flux prediction at SK is shown in Fig. 1 for each neutrino type and for both neutrino- and antineutrino-mode beams. At the peak energy of the T2K beam, the $\nu_{\mu}$ flux in the neutrino-mode beam is $20 \%$ higher

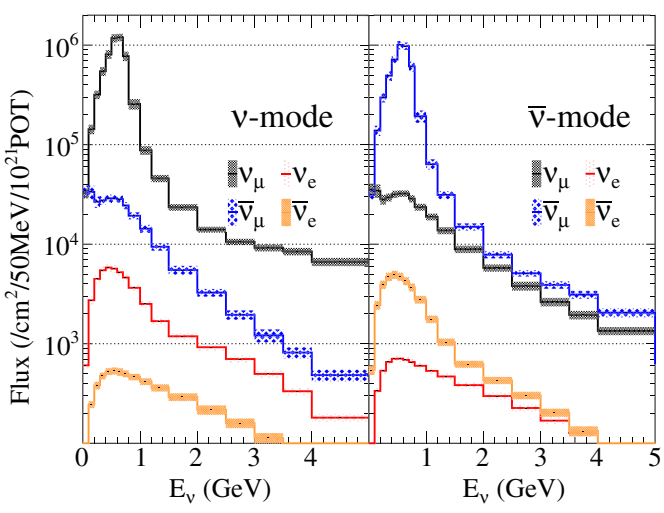

FIG. 1. The nominal unoscillated neutrino flux prediction at SK for each neutrino type in the neutrino-mode beam (left) and antineutrino-mode beam (right). The shaded boxes indicate the total systematic uncertainty on each energy bin.

than the $\bar{\nu}_{\mu}$ flux in the antineutrino-mode beam, due to the larger production cross section for $\pi^{+}$compared to $\pi^{-}$in proton-carbon interactions. The ratio of the wrong-sign component $\left(\nu_{\mu}\right.$ in the $\bar{\nu}_{\mu}$ beam), mainly coming from forward-going high-energy pions, to the right-sign component $\left(\bar{\nu}_{\mu}\right)$ at the peak energy is $3 \%$. The largest sources of neutrino flux uncertainty are from beam-line and hadronproduction modeling uncertainties, which are common to ND280 and SK. The new NA61/SHINE 2009 thin-target data [19] are included in the hadron-production tuning for this analysis, reducing the total flux uncertainty from between $12 \%-15 \%$ to $10 \%$ around $0.6 \mathrm{GeV}$.

Neutrino interaction simulation.-Neutrino interactions are modeled with the NEUT Monte Carlo event generator [20-24]. The generator uses the same model with common parameters to describe both $\nu$ and $\bar{\nu}$ interactions. In the case of CC quasielastic (CCQE) reactions $\left(\nu_{\mu}+n \rightarrow \mu^{-}+p\right.$ or $\left.\bar{\nu}_{\mu}+p \rightarrow \mu^{+}+n\right)$ neutrino and antineutrino cross sections differ by the sign of the vector-axial interference term $[25,26]$. At a neutrino energy of $0.6 \mathrm{GeV}$, this makes the neutrino-oxygen CCQE cross section a factor of $\sim 4$ larger than that of antineutrinos.

To set the initial values and uncertainties of some parameters, such as the CCQE axial mass and the normalization of the multinucleon contribution, results from the MiniBooNE and MINER $\nu \mathrm{A}$ experiments [27-30] on $\mathrm{CH}_{2}$ and $\mathrm{CH}$ targets are used. These parameters are then tuned by the near-detector fit.

Near-detector fit.-The seven samples used in the near-detector fit are summarized in Table I. Muonneutrino-induced $\mathrm{CC}$ interactions in the neutrino beam mode are found by requiring that the highest-momentum, negative-curvature track in an event starts within the upstream FGD (FGD1) fiducial volume (FV) and has an energy deposit in TPC 2 consistent with a muon. Events with a TPC track that starts upstream of the start point of the muon candidate are rejected, and the remaining $\nu_{\mu} \mathrm{CC}$ candidates are divided into three subsamples according to 
TABLE I. Data and MC-predicted event rates for the different ND280 samples before and after the fit. Errors indicate systematic uncertainties only.

\begin{tabular}{lccc}
\hline \hline Sample & Data & Prefit & Postfit \\
\hline \multicolumn{4}{c}{$\nu$ beam mode } \\
$\nu_{\mu}$ CC $0 \pi$ & 17362 & $15625 \pm 1663$ & $17248 \pm 133$ \\
$\nu_{\mu}$ CC $1 \pi^{+}$ & 3988 & $4748 \pm 686$ & $4190 \pm 60$ \\
$\nu_{\mu}$ CC other & 4219 & $3772 \pm 431$ & $4079 \pm 62$ \\
& $\bar{\nu}$ beam mode \\
$\bar{\nu}_{\mu}$ CC 1 track & 435 & $387 \pm 41$ & $438 \pm 13$ \\
$\bar{\nu}_{\mu}$ CC $N$ tracks & 136 & $128 \pm 17$ & $129 \pm 5$ \\
$\nu_{\mu}$ CC 1 track & 131 & $141 \pm 15$ & $147 \pm 6$ \\
$\nu_{\mu}$ CC $N$ tracks & 145 & $147 \pm 17$ & $144 \pm 6$ \\
\hline \hline
\end{tabular}

the number of associated pions: $\nu_{\mu} \mathrm{CC} 0 \pi, \nu_{\mu} \mathrm{CC} 1 \pi^{+}$, and $\nu_{\mu} \mathrm{CC}$ other, which are dominated by CCQE, CC resonant pion production, and deep inelastic scattering interactions, respectively [13]. For the antineutrino-beam-mode samples, the selection of $\bar{\nu}_{\mu}\left(\nu_{\mu}\right)$ CC interactions is similar to that used in the neutrino beam mode, except the positive (negative) track must be the highest-momentum track in the event. The selected $\bar{\nu}_{\mu}\left(\nu_{\mu}\right)$ CC candidate events are divided into two subsamples rather than three, due to the small amount of antineutrino-mode data used in this analysis. These are defined by the number of reconstructed tracks crossing TPC2: $\bar{\nu}_{\mu}\left(\nu_{\mu}\right)$ CC 1 track, dominated by CCQE interactions; and $\bar{\nu}_{\mu}\left(\nu_{\mu}\right) \mathrm{CC} N$ tracks $(N>1)$, a mixture of resonant production and deep inelastic scattering.

The fit uses a binned likelihood, with the samples binned according to the muon momentum and angle $(\theta)$ relative to the central axis of the detector, roughly $1.7^{\circ}$ away from the incident (anti)neutrino direction. The TPCs calculate the muon momentum from the curvature of the lepton in the ND280 magnetic field, with a resolution of $6 \%$ at $1 \mathrm{GeV} / c$ [9]. Figure 2 shows the 1D projections of these distributions for the $\nu_{\mu} \mathrm{CC} 0 \pi$, the $\bar{\nu}_{\mu} \mathrm{CC} 1$ track, and the $\nu_{\mu}$ CC 1 track samples for both data and the post-fit MC model. The $p$-value of the data fit likelihood ratio was found to be 0.05 , and the agreement between the ND280 data and the MC model was judged to be acceptable. The fit gives estimates for 25 antineutrino beam flux parameters at SK, 12 cross-section parameters (including 4 specific to oxygen), and their covariance. There are also additional parameters to control pion final state interactions (FSI) and reinteractions within the detector, which are independent for ND280 and SK.

To decouple the properties of the carbon target at ND280 from those of the oxygen target at SK, separate Fermi momentum, binding energy, multinucleon event normalization, and $\mathrm{CC}$ coherent pion-production normalization parameters are introduced for interactions on oxygen. Since oxygen comprises only $3.6 \%$ by mass of the FGD1 target, this near-detector analysis is insensitive to
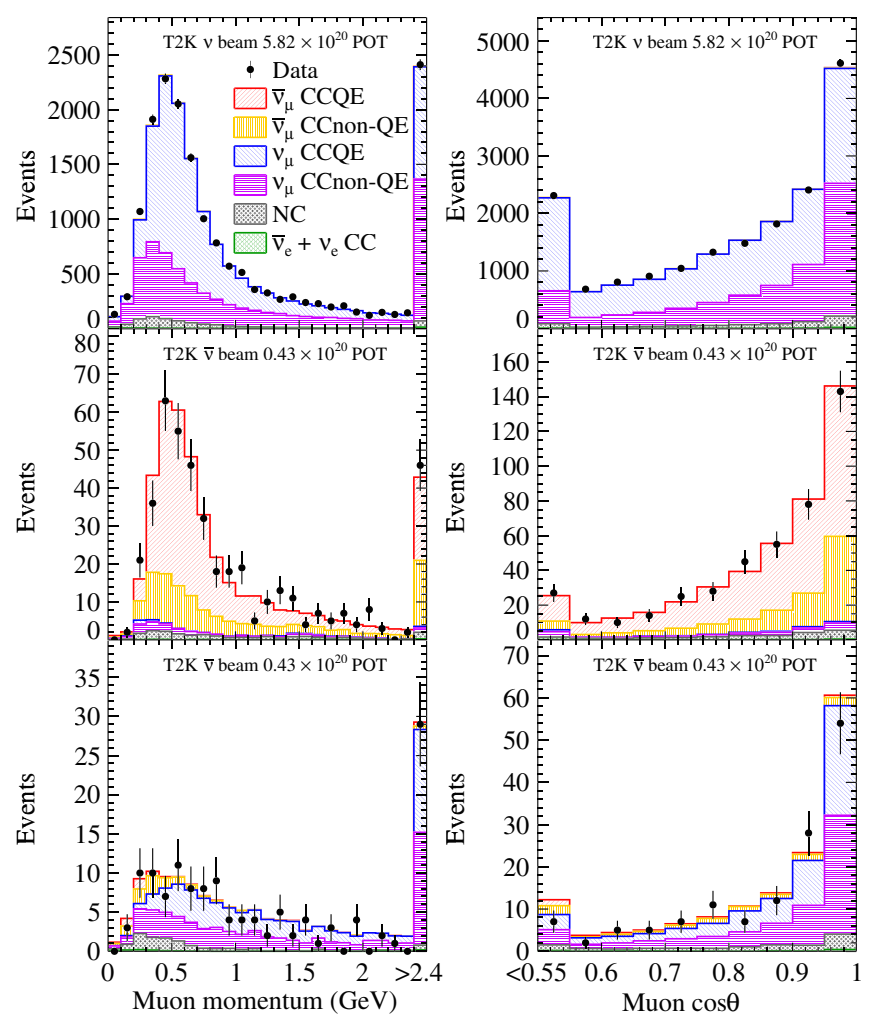

FIG. 2. The momentum (left) and angular (right) distributions of the muon candidates at ND280 from the $\nu_{\mu} \mathrm{CC} 0 \pi$ (top), the $\bar{\nu}_{\mu}$ CC 1 track (center) and the $\nu_{\mu}$ CC 1 track (bottom) samples. The data are superimposed on the post-fit MC prediction, separated by interaction mode.

these parameters. A conservative ( $100 \%$ uncertainty) ansatz is adopted for the normalization of multinucleon ejection oxygen events, giving a $9.5 \%$ uncertainty on the number of events at SK. For the parameters that ND280 can constrain, the fit reduces their effect on the uncertainty on the expected number of events at SK from $9.2 \%$ to $3.4 \%$.

Far-detector selection.-At the far detector, fully contained fiducial volume (FCFV) events are selected by requiring no hit clusters in the OD, that the reconstructed interaction vertex is more than $2 \mathrm{~m}$ away from the ID wall, and that the visible energy in the event is larger than $30 \mathrm{MeV}$. The last criterion requires that the amount of Cherenkov light is more than that of a $30-\mathrm{MeV}$ electromagnetic shower.

To enhance the $\bar{\nu}_{\mu}$ CCQE purity of the sample, selected events must have a single, $\mu$-like Cherenkov ring, no more than one decay electron, and a muon momentum greater than $0.2 \mathrm{GeV}$ [13]. The number of data and $\mathrm{MC}$ events passing each selection criterion are shown in Table II and the reconstructed energy spectrum of the 34 selected events is plotted in Fig. 3. The reconstructed neutrino energy is calculated using the muon momentum and production angle, under the assumption that a CCQE interaction occurred on a nucleon at rest. The selection efficiency for $\bar{\nu}_{\mu} \mathrm{CCQE}$ is estimated to be $77 \%$ while backgrounds 
TABLE II. The number of events observed at the far detector in the antineutrino-beam-mode data after applying each selection cut. MC expectation is calculated assuming oscillations with $\sin ^{2}\left(\theta_{23}\right)=\sin ^{2}\left(\bar{\theta}_{23}\right)=0.5, \quad\left|\Delta m_{32}^{2}\right|=\left|\Delta \bar{m}_{32}^{2}\right|=$ $2.4 \times 10^{-3} \mathrm{eV}^{2}$, and $\sin ^{2}\left(\theta_{13}\right)=\sin ^{2}\left(\bar{\theta}_{13}\right)=0.0257$. The " $\bar{\nu}_{e}+\nu_{e}+\mathrm{NC}$ " column includes the $\mathrm{NC}$ interactions of all the (anti)neutrino flavors. Efficiency numbers are calculated with respect to the number of $\mathrm{MC}$ events generated in the fiducial volume (FV interaction).

\begin{tabular}{lcrrrrrr}
\hline \hline & \multicolumn{9}{c}{ Total } & \multicolumn{3}{c}{ CCQE } & \multicolumn{2}{c}{ CCnonQE } \\
& Data & \multicolumn{1}{c}{ MC } & \multicolumn{1}{c}{$\bar{\nu}_{\mu}$} & \multicolumn{1}{c}{$\nu_{\mu}$} & \multicolumn{1}{c}{$\bar{\nu}_{\mu}$} & \multicolumn{1}{c}{$\nu_{\mu}$} & $\bar{\nu}_{e}+\nu_{e}+\mathrm{NC}$ \\
\hline FV interaction & $\cdots$ & 186.7 & 17.8 & 11.4 & 20.0 & 36.5 & 101 \\
FCFV & 90 & 99.7 & 14.4 & 8.6 & 15.1 & 26.6 & 35.1 \\
Single ring & 50 & 52.2 & 14.0 & 7.7 & 8.1 & 8.7 & 13.8 \\
$\mu$-like & 40 & 39.4 & 13.8 & 7.6 & 7.8 & 8.0 & 2.2 \\
$P_{\mu}>0.2 \mathrm{GeV}$ & 40 & 39.3 & 13.8 & 7.6 & 7.8 & 8.0 & 2.2 \\
$N_{\text {decay- } e}<2$ & 34 & 36.1 & 13.7 & 7.5 & 7.3 & 5.6 & 2.1 \\
Efficiency (\%) & & & 77.1 & 65.7 & 36.6 & 15.3 & 2.0 \\
\hline \hline
\end{tabular}

from neutral-current $(\mathrm{NC}), \nu_{e}$, and $\bar{\nu}_{e}$ interactions are reduced by a factor of 50 . The systematic uncertainties in the detector response are evaluated using atmospheric neutrinos, cosmic-ray muons, and their decay electrons [13].

Oscillation fit.-The oscillation parameters $\sin ^{2}\left(\bar{\theta}_{23}\right)$ and $\Delta \bar{m}_{32}^{2}$ are estimated using a maximum-likelihood fit to the measured reconstructed energy spectrum in the far detector. All other oscillation parameters are fixed as shown in Table III. Oscillation probabilities are calculated using the full three-flavor oscillation framework [31], assuming the normal mass hierarchy $\left(\Delta m_{32}^{2}>0\right)$. Matter effects are included with an Earth density of $\rho=2.6 \mathrm{~g} / \mathrm{cm}^{3}$ [32].

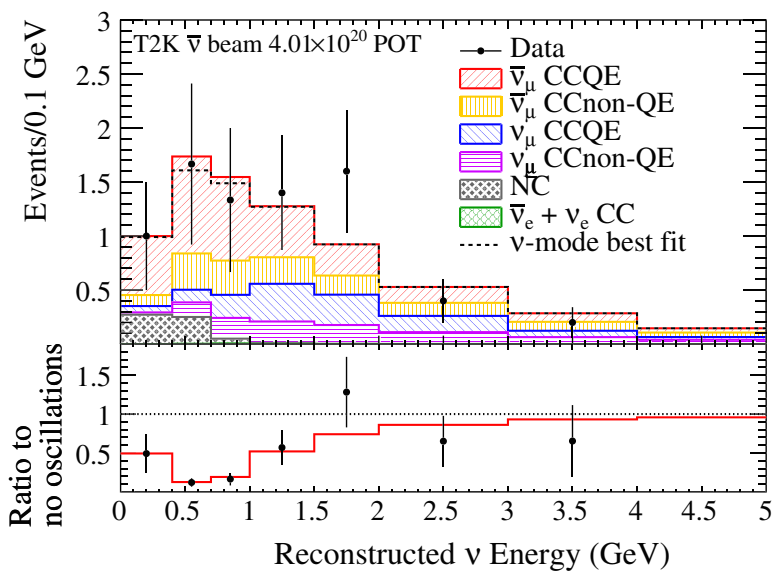

FIG. 3. Top: The reconstructed energy distribution of the 34 far-detector $\bar{\nu}_{\mu}$ candidates and the best-fit prediction, separated by interaction mode. This is compared to the predicted spectrum assuming the antineutrino oscillation parameters are identical to the neutrino parameters measured by T2K [13]. Bottom: The observed data and $\bar{\nu}_{\mu}$-mode best-fit prediction as a ratio to the unoscillated prediction.
TABLE III. Oscillation parameters used for the fit. The parameters $\sin ^{2}\left(\bar{\theta}_{23}\right)$ and $\Delta \bar{m}_{32}^{2}$ were allowed to fit in the ranges given. All other parameters were fixed to the values shown, taken from previous T2K fits [13] and the Particle Data Group review [33].

\begin{tabular}{|c|c|c|}
\hline Parameter & $\nu$ & $\bar{\nu}$ \\
\hline $\sin ^{2}\left(\theta_{23}\right)$ & 0.527 & fit $0-1$ \\
\hline$\Delta m_{32}^{2}\left(\times 10^{-3} \mathrm{eV}^{2}\right)$ & 2.51 & fit $0-20$ \\
\hline $\sin ^{2}\left(\theta_{13}\right)$ & \multicolumn{2}{|c|}{0.0248} \\
\hline $\sin ^{2}\left(\theta_{12}\right)$ & \multicolumn{2}{|c|}{0.304} \\
\hline$\Delta m_{21}^{2}\left(\times 10^{-5} \mathrm{eV}^{2}\right)$ & \multicolumn{2}{|c|}{7.53} \\
\hline$\delta_{C P}(\mathrm{rad})$ & \multicolumn{2}{|c|}{-1.55} \\
\hline
\end{tabular}

Confidence regions are constructed for the oscillation parameters using the constant $\Delta \chi^{2}$ method [33]. A marginal likelihood is used for this, integrating over the nuisance parameters $\mathbf{f}$ with prior probability functions $\pi(\mathbf{f})$ to find the likelihood as a function of only the relevant oscillation parameters $\mathbf{0}$,

$$
\mathcal{L}(\mathbf{o})=\int \prod_{i}^{\text {Ebins }} \mathcal{L}_{i}(\mathbf{o}, \mathbf{f}) \times \pi(\mathbf{f}) d \mathbf{f},
$$

where Ebins denotes the number of reconstructed neutrino energy bins.

We define $\Delta \chi^{2}=-2 \ln [\mathcal{L}(\mathbf{0}) / \max (\mathcal{L})]$ as the ratio of the marginal likelihood at a point $\mathbf{o}$ in the $\sin ^{2}\left(\bar{\theta}_{23}\right)-\Delta \bar{m}_{32}^{2}$ oscillation parameter space and the maximum marginal likelihood. The confidence region is then defined as the area of the oscillation parameter space for which $\Delta \chi^{2}$ is less than a standard critical value. The Feldman-Cousins critical chi-square value was calculated for a coarse set of points in the oscillation parameter space. The difference in the confidence region calculated from these points and that from the standard chi-square values was found to be negligible.

Table IV summarizes the fractional error on the expected number of SK events from a $1 \sigma$ variation of the flux, crosssection, and far-detector systematic parameters. Although the fractional error on the expected number of events due to

TABLE IV. Percentage change in the number of one-ring $\mu$-like events before the oscillation fit from $1 \sigma$ systematic parameter variations, assuming the oscillation parameters listed in Table III and that the antineutrino and neutrino oscillation parameters are identical.

\begin{tabular}{lc}
\hline \hline Source of uncertainty (number of parameters) & $\delta n_{\mathrm{SK}}^{\exp } / n_{\mathrm{SK}}^{\exp }(\%)$ \\
\hline ND280-unconstrained cross section (6) & 10.0 \\
Flux and ND280-constrained cross section (31) & 3.4 \\
Super-Kamiokande detector systematics (6) & 3.8 \\
Pion FSI and reinteractions (6) & 2.1 \\
Total (49) & 11.6 \\
\hline \hline
\end{tabular}




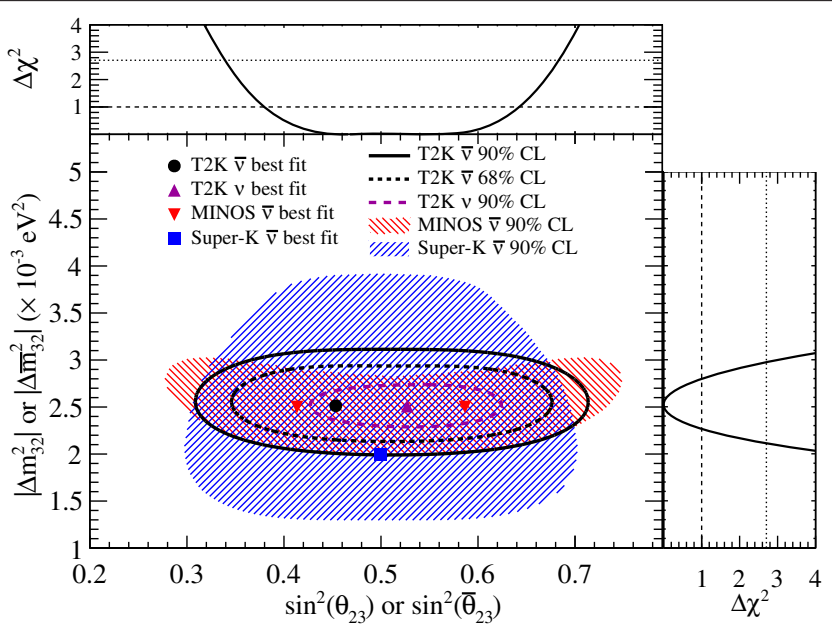

FIG. 4. The $68 \%$ and $90 \%$ confidence regions for the $\sin ^{2}\left(\bar{\theta}_{23}\right)-\left|\Delta \bar{m}_{32}^{2}\right|$ plane assuming normal hierarchy, alongside the T2K $\nu$ [13], SK Collaboration $\bar{\nu}$ [5], and MINOS $\bar{\nu}$ [4] $90 \%$ confidence regions. One-dimensional $\Delta \chi^{2}$ profiles for the two parameters are shown at the top and right, overlaid with lines representing the 1D $\Delta \chi^{2}$ values for the $68 \%$ and $90 \%$ confidence intervals.

systematic errors is large, the effect of systematic parameters on the confidence regions found in this fit is negligible due to the limited data statistics. The impact of fixing the values of $\sin ^{2}\left(\theta_{23}\right)$ and $\Delta m_{32}^{2}$ in the fit is also negligible.

The observed $\bar{\nu}_{\mu}$ reconstructed energy spectrum from the antineutrino-beam-mode data is shown in the upper plot of Fig. 3, overlaid with the best-fit spectrum assuming normal hierarchy, separated by interaction mode. The lower plot in Fig. 3 is the ratio of data to the expected, unoscillated spectrum.

The best-fit values obtained are $\sin ^{2}\left(\bar{\theta}_{23}\right)=0.45$ and $\left|\Delta \bar{m}_{32}^{2}\right|=2.51 \times 10^{-3} \mathrm{eV}^{2}$, with $68 \%$ confidence intervals of $0.38-0.64$ and $2.26-2.80 \times 10^{-3} \mathrm{eV}^{2}$, respectively. A goodness-of-fit test was performed by comparing this fit to an ensemble of toy experiments, giving a $p$-value of 0.38 .

The fit results are shown in Fig. 4 as $68 \%$ and $90 \%$ confidence regions in the $\sin ^{2}\left(\bar{\theta}_{23}\right)-\Delta \bar{m}_{32}^{2}$ plane. The $90 \%$ confidence regions from the T2K neutrino-beammode joint disappearance and appearance fit [13], the SK fit to $\bar{\nu}_{\mu}$ in atmospheric neutrino data [5], and the MINOS fit to $\bar{\nu}_{\mu}$ beam and atmospheric data [4] are also shown for comparison. A second, fully Bayesian, analysis was also performed, producing a credible region matching the confidence regions presented above.

Conclusions. - We report the first study of $\bar{\nu}_{\mu}$ disappearance using an off-axis beam and present measurements of $\sin ^{2}\left(\bar{\theta}_{23}\right)=0.45$ and $\Delta \bar{m}_{32}^{2}=2.51 \times 10^{-3} \mathrm{eV}^{2}$. These results are consistent with the values of $\sin ^{2}\left(\theta_{23}\right)$ and $\Delta m_{32}^{2}$ observed previously by $\mathrm{T} 2 \mathrm{~K}$ [13], providing no indication of new physics, and are also in good agreement with similar measurements from MINOS [4] and the SK
Collaboration [5]. The results presented here, with the first T2K antineutrino data set, are competitive with those from both the MINOS and SK Collaborations, demonstrating the effectiveness of the off-axis beam technique.

We thank the J-PARC staff for superb accelerator performance and the CERN NA61 Collaboration for providing valuable particle production data. We acknowledge the support of MEXT, Japan; NSERC (Grant No. SAPPJ-2014-00031), NRC and CFI, Canada; CEA and CNRS/IN2P3, France; DFG, Germany; INFN, Italy; National Science Centre (NCN), Poland; RSF, RFBR, and MES, Russia; MINECO and ERDF funds, Spain; SNSF and SERI, Switzerland; STFC, UK; and DOE, USA. We also thank CERN for the UA1/NOMAD magnet, DESY for the HERA-B magnet mover system, NII for SINET4, the WestGrid and SciNet consortia in Compute Canada, and GridPP and the Emerald High Performance Computing facility in the United Kingdom. In addition, participation of individual researchers and institutions has been further supported by funds from ERC (FP7), H2020 Grant No. RISE-GA644294-JENNIFER, EU; JSPS, Japan; Royal Society, UK; and the DOE Early Career program, USA.

\footnotetext{
*Deceased.

Also at J-PARC, Tokai, Japan.

${ }^{\sharp}$ Also at Kavli IPMU (WPI), University of Tokyo, Japan.

§Also at National Research Nuclear University "MEPhI" and Moscow Institute of Physics and Technology, Moscow, Russia.

"Also at JINR, Dubna, Russia.

"Also at Institute of Particle Physics, Canada.

** Also at BMCC/CUNY, Science Department, New York, New York, USA.
}

[1] Z. Maki, M. Nakagawa, and S. Sakata, Prog. Theor. Phys. 28, 870 (1962).

[2] B. Pontecorvo, Zh. Eksp. Teor. Fiz. 53, 1717 (1967) [Sov. Phys. JETP 26, 984 (1968)].

[3] J. Beringer et al. (Particle Data Group), Phys. Rev. D 86, 010001 (2012).

[4] P. Adamson et al. (MINOS Collaboration), Phys. Rev. Lett. 108, 191801 (2012).

[5] K. Abe et al. (Super-Kamiokande Collaboration), Phys. Rev. Lett. 107, 241801 (2011).

[6] K. Abe et al. (T2K Collaboration), Nucl. Instrum. Methods Phys. Res., Sect. A 659, 106 (2011).

[7] D. Beavis, A. Carroll, I. Chiang et al. (E889 Collaboration), Brookhaven National Laboratory Report No. 52459, 1995.

[8] K. Abe et al. (T2K Collaboration), Nucl. Instrum. Methods Phys. Res., Sect. A 694, 211 (2012).

[9] N. Abgrall et al. (T2K ND280 TPC Collaboration), Nucl. Instrum. Methods Phys. Res., Sect. A 637, 25 (2011).

[10] P. Amaudruz et al. (T2K ND280 FGD Collaboration), Nucl. Instrum. Methods Phys. Res., Sect. A 696, 1 (2012).

[11] S. Fukuda et al. (Super-Kamiokande Collaboration), Nucl. Instrum. Methods Phys. Res., Sect. A 501, 418 (2003). 
[12] K. Abe et al. (Super-Kamiokande Collaboration), Nucl. Instrum. Methods Phys. Res., Sect. A 737, 253 (2014).

[13] K. Abe et al. (T2K Collaboration), Phys. Rev. D 91, 072010 (2015).

[14] K. Abe et al. (T2K Collaboration), Phys. Rev. D 87, 012001 (2013); 87, 019902 (2013).

[15] A. Ferrari, P. R. Sala, A. Fasso, and J. Ranft, Reports No. CERN-2005-010, SLAC-R-773, and INFN-TC-0511, 2005.

[16] T. T. Böhlen, F. Cerutti, M. P. W. Chin, A. Fassò, A. Ferrari, P. G. Ortega, A. Mairani, P. R. Sala, G. Smirnov, and V. Vlachoudis, Nucl. Data Sheets 120, 211 (2014).

[17] R. Brun, F. Carminati, and S. Giani, Report No. CERNW5013, 1994.

[18] C. Zeitnitz and T. A. Gabriel, in Proceedings of International Conference on Calorimetry in High Energy Physics, Tallahassee, FL, USA, February 1993 (World Scientific, Singapore, 2014).

[19] N. Abgrall et al. (NA61/SHINE Collaboration), Eur. Phys. J. C 76, 84 (2016).

[20] Y. Hayato, Acta Phys. Pol. B 40, 2477 (2009).

[21] Version 5.3.2 of the NEUT library is used, which includes (i) the multinucleon ejection model of Nieves et al. [22] and (ii) nuclear long-range correlations for CCQE interactions, treated in the random phase approximation [23].
[22] J. Nieves, I. R. Simo, and M. J. V. Vacas, Phys. Rev. C 83, 045501 (2011).

[23] J. Nieves, J. E. Amaro, and M. Valverde, Phys. Rev. C 70, 055503 (2004); 72, 019902(E) (2005).

[24] C. Wilkinson, in Proceedings of 16th International Workshop on Neutrino Factories and Future Neutrino Beam Facilities (NUFACT 2014), (Glasgow, Scotland, UK, August 2014).

[25] C. H. Llewellyn Smith, Phys. Rep. 3, 261 (1972).

[26] M. Jacob, Gauge Theories and Neutrino Physics (North-Holland, Amsterdam, 1978).

[27] A. A. Aguilar-Arevalo et al. (MiniBooNE Collaboration), Phys. Rev. D 81, 092005 (2010).

[28] A. A. Aguilar-Arevalo et al. (MiniBooNE Collaboration), Phys. Rev. D 88, 032001 (2013).

[29] G. Fiorentini et al. (MINERvA Collaboration), Phys. Rev. Lett. 111, 022502 (2013).

[30] L. Fields et al. (MINERvA Collaboration), Phys. Rev. Lett. 111, 022501 (2013).

[31] V. Barger, K. Whisnant, S. Pakvasa, and R. J. N. Phillips, Phys. Rev. D 22, 2718 (1980).

[32] K. Hagiwara, N. Okamura, and K. Senda, J. High Energy Phys. 11 (2011) 082.

[33] K. A. Olive et al. (Particle Data Group), Chin. Phys. C 38, 090001 (2014). 